\title{
Influence of Conversion Coating on Magnesium and Aluminum Alloys by Adhesion Method
}

\author{
Manabu Takai* and Matsufumi Takaya \\ Department of Mechanical Science and Engineering, Chiba Institute of Technology, Narashino 275-0016, Japan
}

\begin{abstract}
Magnesium alloys are lightweight materials with high specific strength and have electromagnetic shielding characteristics. On the other hand, chromate is known as carcinogen and it leads a limited use of the conversion coating on magnesium. In recent years, adhesion technology is essential and widely used in producing electronic equipment and sporting goods from magnesium alloys. Adhesives can easily bind different materials. Adhesives may even inhibit the contact corrosion of magnesium, and we may expect adhesion to be used to effectively join different metals.

This paper describes and compared with the influence of various non-chromate conversion coatings on the bonding strength of magnesium and aluminum alloys.

In this study, specimens of AZ31B and A2017, A6022 were used. The adhesive cements used were epoxy and acrylic resin. The results of a pull-off test and tensile lap-shear strength test ware evaluated.

The $\mathrm{Mg}(\mathrm{OH})_{2}$ coating on AZ31B and Boehmite on A6022 exhibited a higher tensile shear strength (16 MPa) with epoxy resin. In the acrylic resin, $\mathrm{Mg}(\mathrm{OH})_{2}$ coating on AZ31B and Boehmite on A6022 had a tensile shear strength of $14 \mathrm{MPa}$.

It is obvious that these conversion coatings can be alternatives to chromate conversion coatings as surface treatment for adhesion of magnesium alloys and aluminum alloys. [doi:10.2320/matertrans.MC2007104]
\end{abstract}

(Received October 19, 2007; Accepted February 15, 2008; Published April 9, 2008)

Keywords: magnesium alloy, aluminum alloy, conversion coating, adhesion method, bond strength

\section{Introduction}

Magnesium alloys are lightweight materials with high specific strength and have electromagnetic shield characteristics. On the other hand, chromate is known as carcinogen and it leads a limited use of the conversion coating on magnesium. ${ }^{1)}$ In recent years, adhesion technology is essential and widely used in producing electronic equipment and sporting goods from magnesium alloys. ${ }^{2)}$ Adhesion can bond different materials easily. Adhesion materials may even inhibit contact corrosion of magnesium, and we may expect adhesion to be used in the effective joining of different metals. We previously reported magnesium alloys bonding with $\mathrm{Mg}(\mathrm{OH})_{2}$ conversion coating. ${ }^{3)}$

This paper describes and compared with the influence of various non-chromate conversion coatings on the bonding strength of magnesium alloy and aluminum alloys.

\section{Experimental Procedure}

The specimens used in this study were AZ31B magnesium alloy, A2017 and A6022 aluminum alloys. Table 1 lists the solution composition and conditions of conversion with magnesium alloy. Table 2 lists solution composition and conditions with aluminum alloys. For pre-treatment of magnesium alloy, the specimen was polished with \#100 emery paper and degreased with 2-propanol. Following this, the specimen was treated by conditions of conversion coating to show in Table 1. Aluminum alloys was immersed in 10 mass $\% \mathrm{NaOH}$ for $60 \mathrm{~s}$ at $333 \mathrm{~K}$, desmutting $10 \mathrm{vol} \%$ $\mathrm{HNO}_{3}$ solution for $15 \mathrm{~s}$ at $293 \mathrm{~K}$. After pre-treatment, the specimen was treated by conditions of conversion coating to show in Table 2 . These specimens were bonded immediately

*Graduate Student, Chiba Institute of Technology after drying. Table 3 presents the adhesive conditions. The adhesive cements used were epoxy and acrylic resin. The bond strength was measured by an adhesion tester. The tensile lap-shear strength was measured by a tensile test machine. Figure 1 depicts a test piece for the pull-off test (JIS K 5600-5-7); Figure 2 depicts a test piece for the tensile shear strength test (JIS K 6850). The surface of the conversion coatings on magnesium and aluminum alloys was observed by scanning electron microscopy (SEM).

\section{Results and Discussions}

\subsection{Observation of conversion coating surfaces}

Figure 3 presents SEM images of various conversion coatings on AZ31B magnesium alloy. The chromate conversion coating was scale and the $\mathrm{Mg}(\mathrm{OH})_{2}$ coating was a thin film. Figure 4 presents SEM images of various conversion coatings on A6022 aluminum alloy. The chromatephosphate conversion coating was scale, the manganese conversion coating was cracked and the boehmite conversion coating was dimple.

\subsection{Evaluation of bond strength by pull-off test ${ }^{4)}$}

Figure 5 illustrates the relationship between bond strength with epoxy resin and various conversion coatings on AZ31B magnesium alloy and A2017 aluminum alloy and, Figure 6 illustrates the relationship between bond strength with acrylic resin and various conversion coatings on AZ31B magnesium alloy and A2017 aluminum alloy. The $\mathrm{Mg}(\mathrm{OH})_{2}$ coating on AZ31B magnesium alloy and boehmite on A2017 aluminum alloy exhibited a higher bond strength $(10 \mathrm{MPa})$ with a $373 \mathrm{~K}$ cure temperature by epoxy resin. In the acrylic resin, $\mathrm{Mg}(\mathrm{OH})_{2}$ coating on AZ31B magnesium alloy and boehmite on A2017 aluminum alloy had a bond strength of $8 \mathrm{MPa}$ with a cure temperature of $293 \mathrm{~K}$. The 
Table 1 Solution composition and condition with magnesium alloy.

\begin{tabular}{|c|c|c|c|c|c|c|}
\hline Specimen & $\begin{array}{c}\text { Polishing \& } \\
\text { Degrease }\end{array}$ & Conversion coating & Composition & $\begin{array}{l}\text { Concentration } \\
\qquad\left(\mathrm{kg} / \mathrm{m}^{3}\right)\end{array}$ & $\begin{array}{c}\text { Temperature } \\
(\mathrm{K})\end{array}$ & $\begin{array}{l}\text { Time } \\
\text { (s) }\end{array}$ \\
\hline \multirow{3}{*}{ AZ31B } & \multirow{3}{*}{$\begin{array}{c}100^{\#} \\
\text { emery paper } \\
\& \\
\text { 2-propanol }\end{array}$} & \multirow{2}{*}{$\begin{array}{c}\text { Chromate } \\
\text { (JIS K 6848-2) }\end{array}$} & $\mathrm{CrO}_{3}$ & 159.0 & & \\
\hline & & & $\mathrm{Ca}\left(\mathrm{NO}_{3}\right)_{2}$ & 13.9 & & \\
\hline & & $\mathrm{Mg}(\mathrm{OH})_{2}$ coating & $\mathrm{NaOH}$ & 80.0 & 343 & 600 \\
\hline
\end{tabular}

Table 2 Solution composition and condition with aluminum alloy.

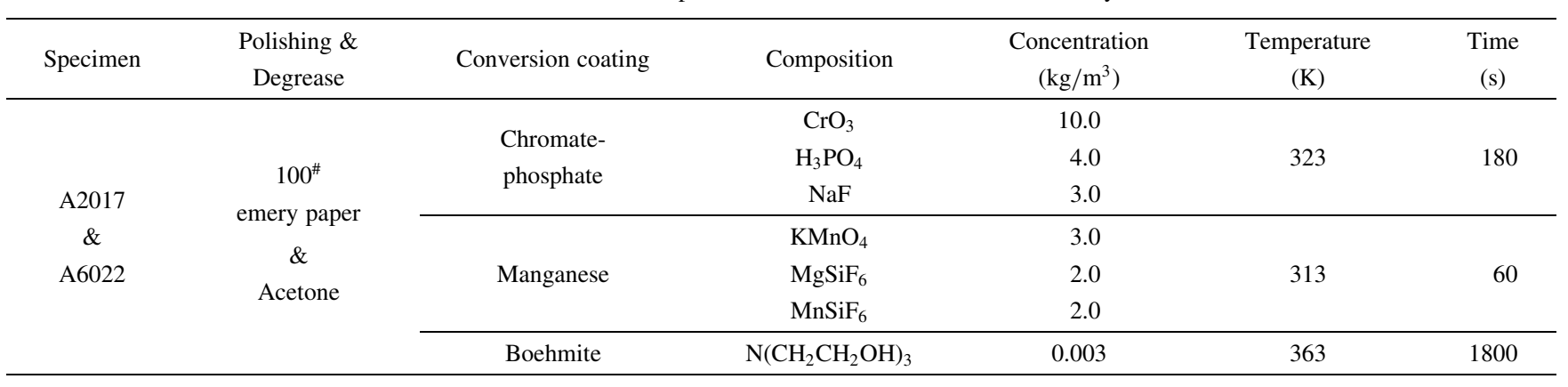

Table 3 Condition of adhesive.

\begin{tabular}{ccc}
\hline Item & \multicolumn{2}{c}{ Condition } \\
\hline Cure temperature $(\mathrm{K})$ & 293 & $323,373,423,473$ \\
\hline Pressing time(hour) & 24 & 1 \\
\hline Pressure $(\mathrm{MPa})$ & \multicolumn{2}{c}{0.06} \\
\hline $\begin{array}{c}\text { Aging(hour) } \\
* \text { Cure temperature }\end{array}$ & $48(293 \mathrm{~K})^{*}$ \\
\hline
\end{tabular}

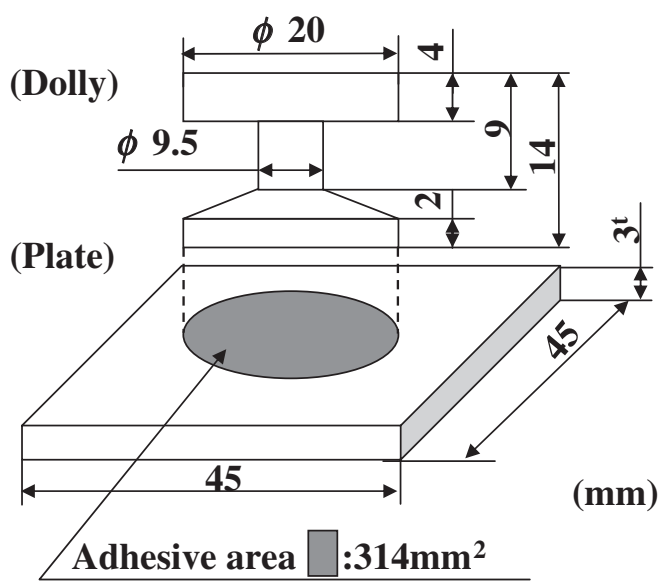

Fig. 1 Test piece of pull-off test.

bond strength of epoxy resin exhibited higher strength with cure temperature at $373 \mathrm{~K}$ and, the bond strength of acrylic resin exhibited higher strength with cure temperature at $293 \mathrm{~K}$.

\subsection{Evaluation of tensile shear strength by tensile lap- shear strength test $^{5)}$}

Figure 7 illustrates the relationship between tensile shear strength with epoxy resin and various conversion coatings

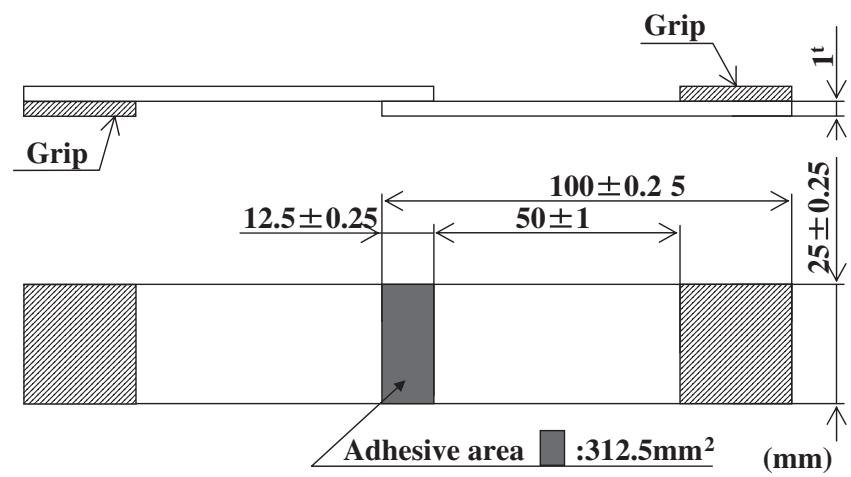

Fig. 2 Test piece of tensile lap-shear test.

on AZ31B magnesium alloy and A6022 aluminum alloy; Figure 8 illustrates the relationship between tensile shear strength with acrylic resin and various conversion coatings on AZ31B magnesium alloy and A6022 aluminum alloy. The $\mathrm{Mg}(\mathrm{OH})_{2}$ coating on AZ31B magnesium alloy and boehmite on A6022 aluminum alloy exhibited a higher tensile shear strength (16 MPa) with epoxy resin. In the acrylic resin, $\mathrm{Mg}(\mathrm{OH})_{2}$ coating on AZ31B magnesium alloy and boehmite on A6022 aluminum alloy had a tensile shear strength of $14 \mathrm{MPa}$.

\subsection{Evaluation of failure patterns ${ }^{6)}$}

Figure 9 shows the model of the failure patterns. The failure patterns were evaluated by adhesive failure (AF), cohesive failure $(\mathrm{CF})$, and delamination failure (DF). Table 4 presents failure patterns with various conversion coatings epoxy resin and acrylic resin (Pull-off test). The results of the pull-off evaluation with epoxy resin indicate cohesive failure $(\mathrm{CF})$ when bond strengths of $\mathrm{Mg}(\mathrm{OH})_{2}$ coating on AZ31B magnesium alloy and boehmite on A2017 aluminum alloy exhibited higher strength with a cure temperature of $373 \mathrm{~K}$. However, a delamination failure 


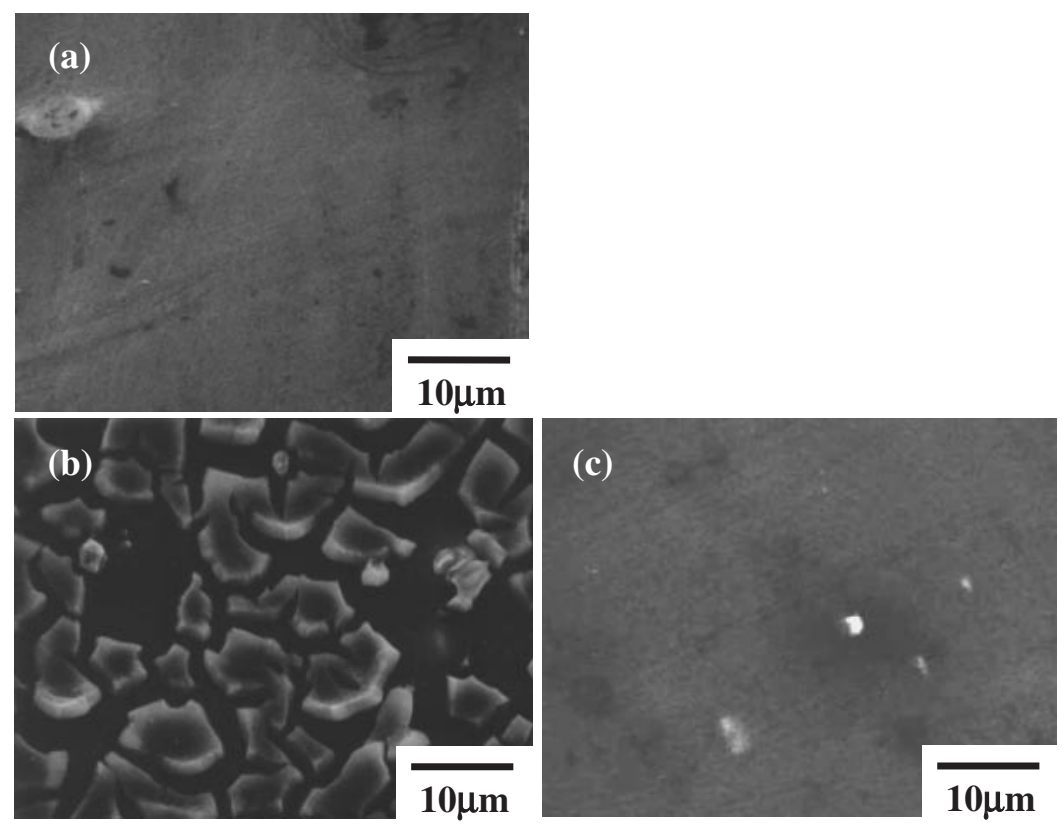

Fig. 3 SEM images of various conversion coatings on AZ31B magnesium alloy. (a) as cleaned, (b) chromate, (c) $\mathrm{Mg}(\mathrm{OH})_{2}$ coating.

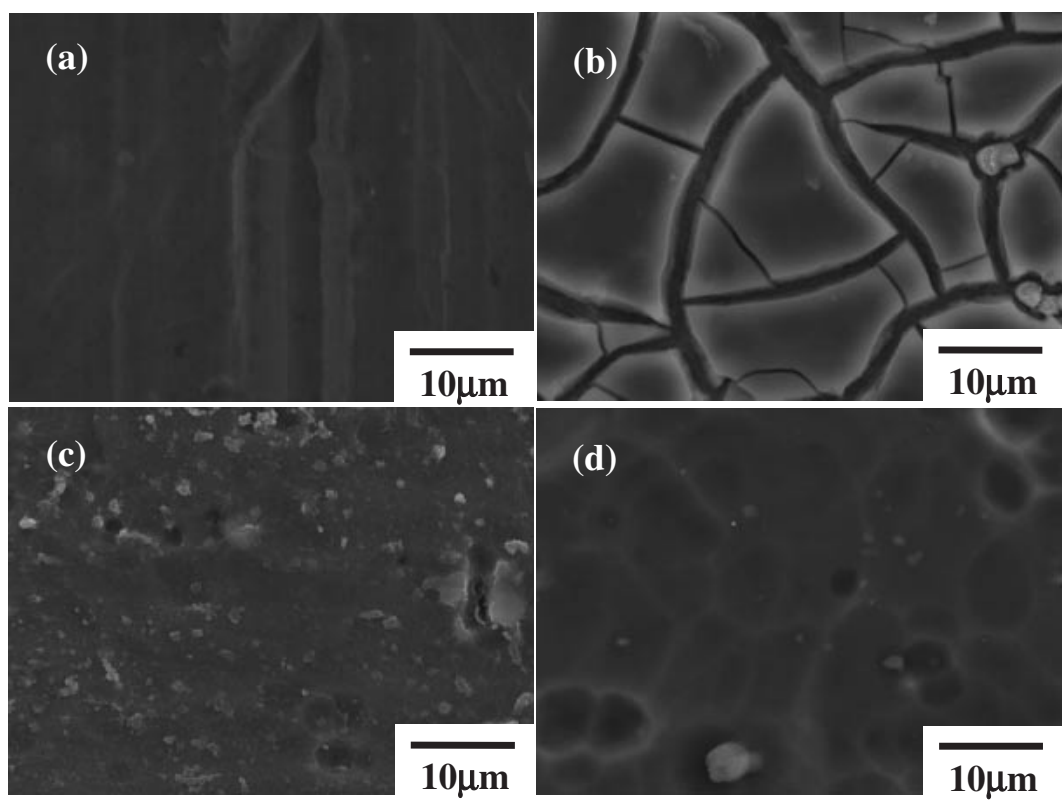

Fig. 4 SEM images of various conversion coatings on A6022 aluminum alloy. (a) as cleaned, (b) chromate-phosphate, (c) manganese, (d) boehmite.

(DF) occurred for chromate on AZ31B magnesium alloy. For the acrylic resin, the failure pattern exhibited cohesive failure $(\mathrm{CF})$ when the bond strengths of as cleaned and $\mathrm{Mg}(\mathrm{OH})_{2}$ coating on AZ31B magnesium alloy were all cure temperatures.

Table 5 presents failure patterns with various conversion coatings epoxy resin and acrylic resin (tensile lap-shear strength test). The results of the tensile lap-shear strength tests with epoxy resin and acrylic resin indicate delamination failure (DF) when tensile shear strengths of chromate on AZ31B magnesium alloy or chromate-phosphate and manganese on A6022 aluminum alloy increased with cure temperature.
Failure patterns were different by various conversion coating. In particular, the chromate coating on AZ31B magnesium alloy and chromate-phosphate coating on aluminum alloys showed delamination failure. We consider that small crack chromate coating showed delamination failure. Therefore, chromate coating and chromate-phosphate coating combination exfoliated all chromate coating side.

\subsection{Observation of galvanic corrosion by salt spray testing ${ }^{7)}$}

The specimens used in this study were AZ31B magnesium alloy and A6022 aluminum alloy; the adhesive cements were used epoxy and acrylic resin. 
(a) 《A2017: As Cleaned》

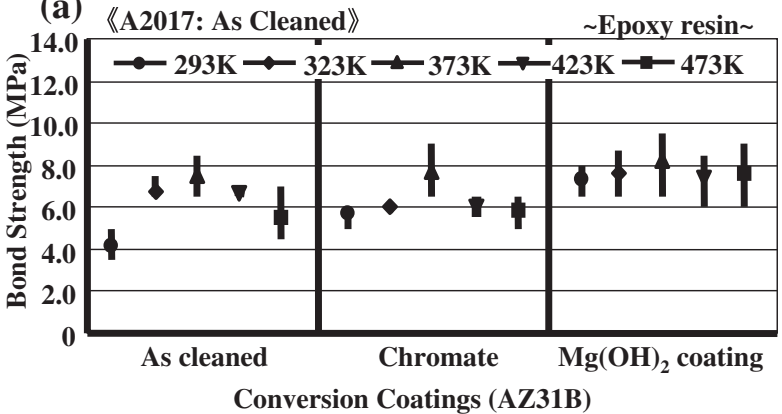

(c)

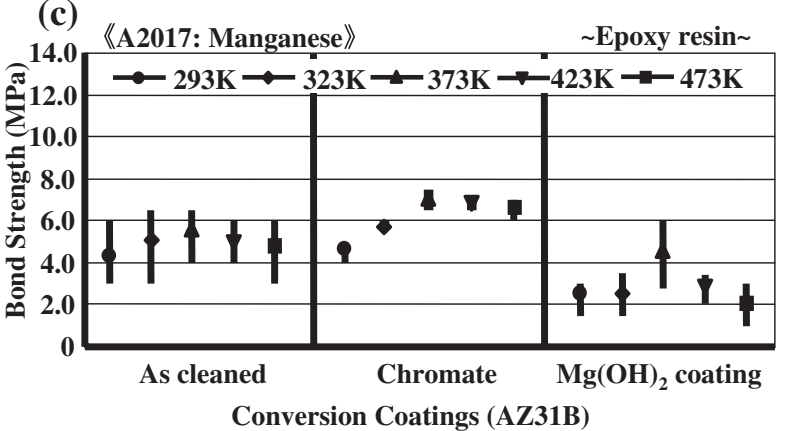

(b)

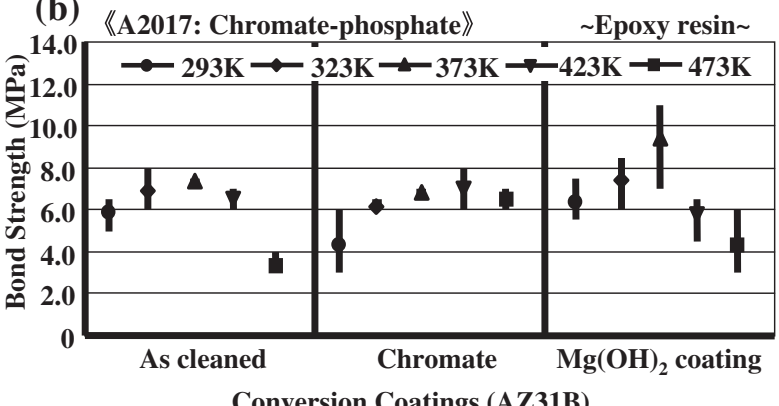

(d)

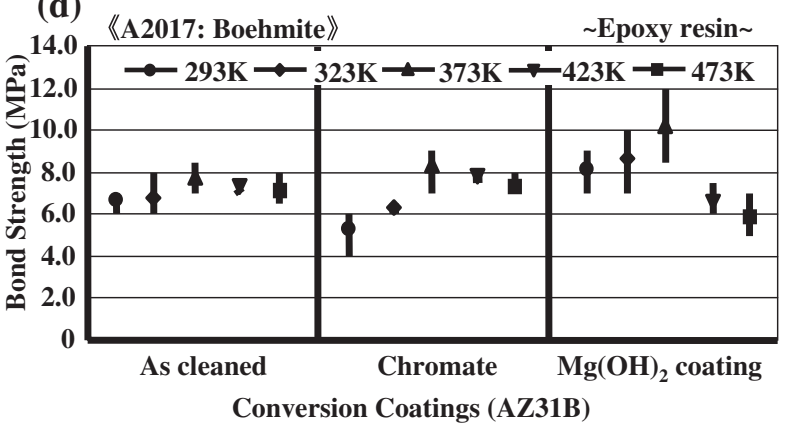

Fig. 5 Relationship between bond strength and various conversion coatings on AZ31B magnesium alloy - A2017 aluminum alloy. (Epoxy resin) A2017 conversion coatings; (a) as cleaned, (b) chromate-phosphate, (c) manganese, (d) boehmite.

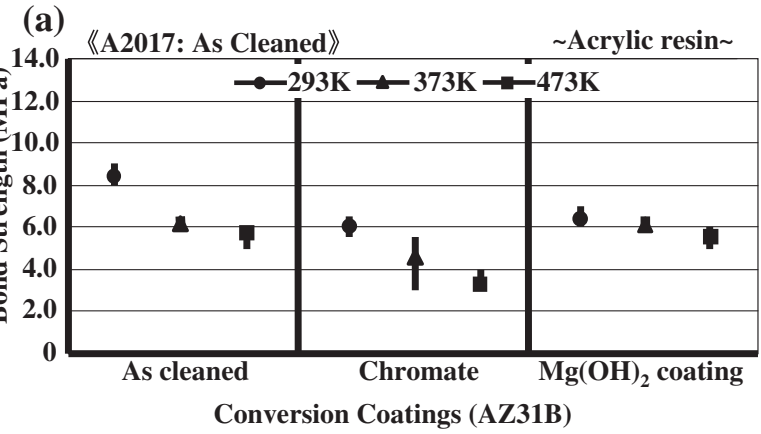

(c)

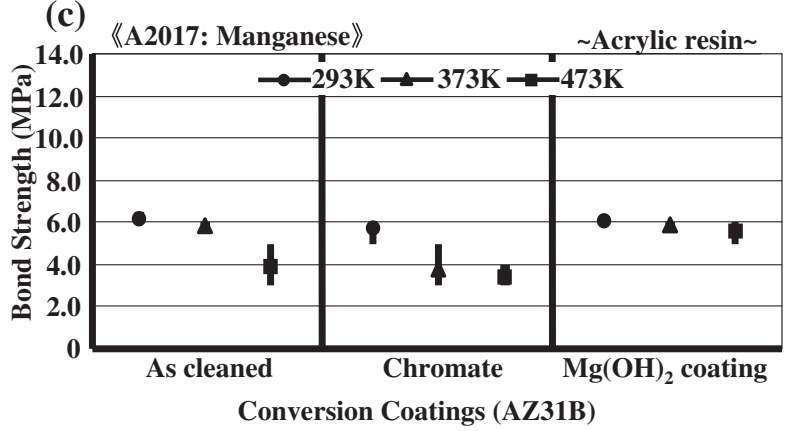

(b)

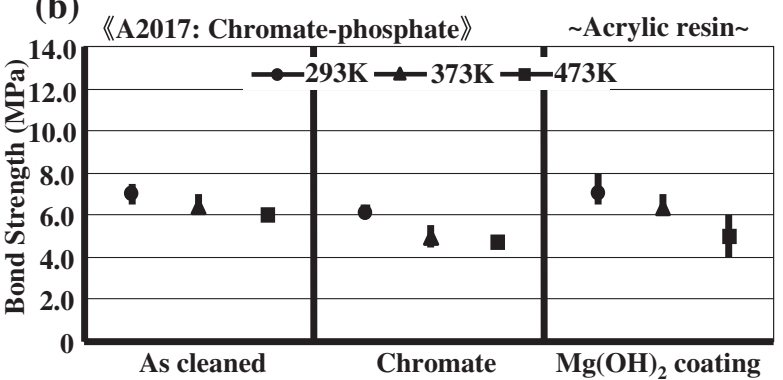

Conversion Coatings (AZ31B)

(d)

Fig. 6 Relationship between bond strength and various conversion coatings on AZ31B magnesium alloy - A2017 aluminum alloy. (Acrylic resin) A2017 conversion coatings; (a) as cleaned, (b) chromate-phosphate, (c) manganese, (d) boehmite.

Figure 10 presents cross section images of galvanic corrosion test. The specimen of without adhesive cement showed in Figure 10(a). Part of corrosion area was larger confirmed in the center of AZ31B magnesium alloy side. Corrosion state of with adhesive cements was not confirmed in the center of AZ31B magnesium alloy side (Figure (b, c)). From above result, the reduction of the galvanic corrosion was able to be confirmed by applying the adhesive cement.

\section{Conclusions}

We found that greater bonding strength than other conversion coatings when using $\mathrm{Mg}(\mathrm{OH})_{2}$ conversion coating on AZ31B magnesium alloy and boehmite conversion coating on A2017 aluminum alloy. In particular, the $\mathrm{Mg}(\mathrm{OH})_{2}$ conversion coating on AZ31B magnesium alloy and Boehmite conversion coating on A6022 aluminum alloy 


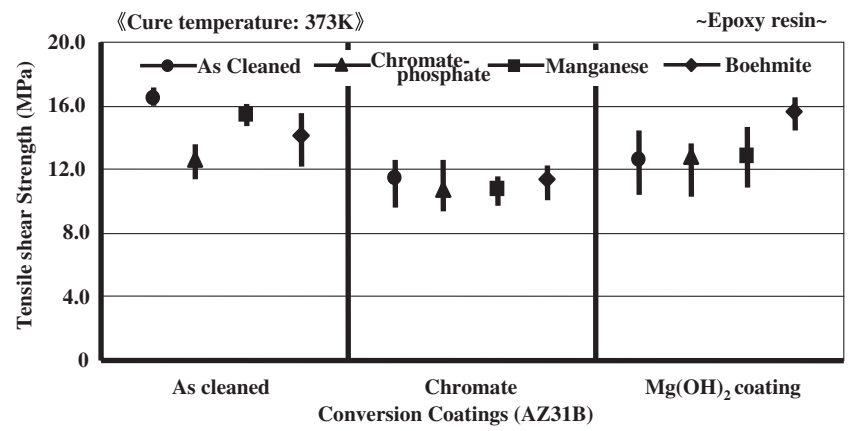

Fig. 7 Relationship between tensile shear strength and various conversion coatings on AZ31B magnesium alloy - A6022 aluminum alloy. (Epoxy resin, Cure temperature: $373 \mathrm{~K}$ ).

had a higher tensile shear strength $(16 \mathrm{MPa})$ when using epoxy resin with a cure temperature of $373 \mathrm{~K}$. The reduction of the galvanic corrosion was able to be confirmed by spreading the adhesive.

It is obvious that these conversion coatings can be alternatives to chromate conversion coatings as surface treatment for adhesion of magnesium alloys and aluminum alloys.

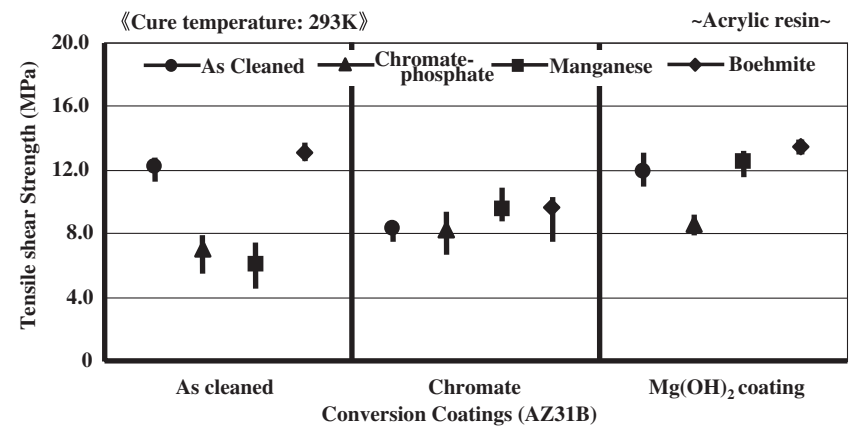

Fig. 8 Relationship between tensile shear strength and various conversion coatings on AZ31B magnesium alloy - A6022 aluminum alloy. (Acrylic resin, Cure temperature: $293 \mathrm{~K}$ ).
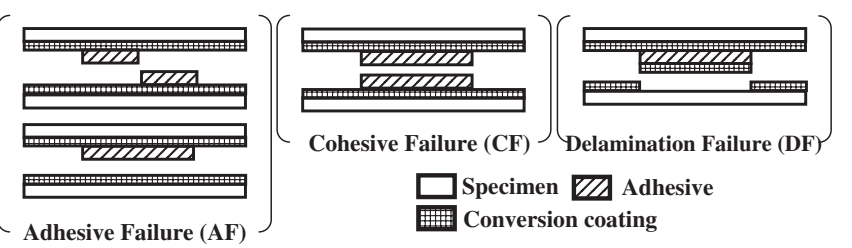

Fig. 9 Model of the failure patterns. (JIS K 6866).

Table 4 Failure patterns with various conversion coatings on AZ31B magnesium alloy - A2017 aluminum alloy. (Pull-off test)

\begin{tabular}{|c|c|c|c|c|c|c|c|c|c|c|c|c|}
\hline \multirow{2}{*}{$\begin{array}{c}\text { Epoxy } \\
\text { resin }\end{array}$} & \multicolumn{2}{|c|}{ Specimen } & \multicolumn{4}{|c|}{ Cure temperature(K) } & \multicolumn{6}{|c|}{ Delamination side } \\
\hline & AZ31B & A2017 & & 293 & & 23 & & 73 & & 23 & & 73 \\
\hline \multirow{12}{*}{$\begin{array}{c}\text { Conversion } \\
\text { coating }\end{array}$} & \multirow{4}{*}{ As cleaned } & As cleaned & $\mathrm{AF}$ & AZ31B & $\mathrm{AF}$ & AZ31B & $\mathrm{AF}$ & AZ31B & $\mathrm{CF}$ & - & $\mathrm{CF}$ & - \\
\hline & & Chromate-phosphate & $\mathrm{AF}$ & A2017 & $\mathrm{AF}$ & A2017 & $\mathrm{AF}$ & A2017 & $\mathrm{CF}$ & - & $\mathrm{CF}$ & - \\
\hline & & Manganese & DF & A2017 & DF & A2017 & DF & A2017 & DF & A2017 & DF & A2017 \\
\hline & & Boehmite & $\mathrm{CF}$ & - & $\mathrm{CF}$ & - & $\mathrm{CF}$ & - & $\mathrm{CF}$ & - & $\mathrm{CF}$ & - \\
\hline & \multirow{4}{*}{ Chromate } & As cleaned & DF & AZ31B & $\mathrm{DF}$ & AZ31B & DF & AZ31B & $\mathrm{DF}$ & AZ31B & $\mathrm{DF}$ & AZ31B \\
\hline & & Chromate-phosphate & DF & AZ31B & $\mathrm{DF}$ & AZ31B & DF & AZ31B & $\mathrm{DF}$ & AZ31B & $\mathrm{DF}$ & AZ31B \\
\hline & & Manganese & DF & AZ31B & $\mathrm{DF}$ & AZ31B & $\mathrm{DF}$ & AZ31B & $\mathrm{DF}$ & AZ31B & $\mathrm{DF}$ & AZ31B \\
\hline & & Boehmite & DF & AZ31B & $\mathrm{DF}$ & AZ31B & DF & AZ31B & DF & AZ31B & DF & AZ31B \\
\hline & \multirow{4}{*}{$\mathrm{Mg}(\mathrm{OH})_{2}$ coating } & As cleaned & $\mathrm{AF}$ & AZ31B & $\mathrm{AF}$ & AZ31B & $\mathrm{AF}$ & AZ31B & $\mathrm{CF}$ & - & $\mathrm{CF}$ & - \\
\hline & & Chromate-phosphate & $\mathrm{AF}$ & A2017 & $\mathrm{AF}$ & A2017 & $\mathrm{AF}$ & A2017 & $\mathrm{CF}$ & - & $\mathrm{CF}$ & - \\
\hline & & Manganese & DF & A2017 & $\mathrm{DF}$ & A2017 & DF & A2017 & $\mathrm{DF}$ & A2017 & DF & A2017 \\
\hline & & Boehmite & $\mathrm{CF}$ & - & $\mathrm{CF}$ & - & $\mathrm{CF}$ & - & $\mathrm{CF}$ & - & $\mathrm{CF}$ & - \\
\hline \multirow{2}{*}{$\begin{array}{l}\text { Acrylic } \\
\text { resin }\end{array}$} & \multicolumn{2}{|c|}{ Specimen } & \multicolumn{4}{|c|}{ Cure temperature(K) } & \multicolumn{6}{|c|}{ Delamination side } \\
\hline & AZ31B & A2017 & \multicolumn{2}{|c|}{293} & \multicolumn{2}{|c|}{323} & \multicolumn{2}{|c|}{373} & \multicolumn{2}{|c|}{423} & \multicolumn{2}{|c|}{473} \\
\hline \multirow{12}{*}{$\begin{array}{c}\text { Conversion } \\
\text { coating }\end{array}$} & \multirow{4}{*}{ As cleaned } & As cleaned & $\mathrm{CF}$ & - & - & - & $\mathrm{CF}$ & - & - & - & $\mathrm{CF}$ & - \\
\hline & & Chromate-phosphate & $\mathrm{CF}$ & - & - & - & $\mathrm{CF}$ & - & - & - & $\mathrm{CF}$ & - \\
\hline & & Manganese & $\mathrm{CF}$ & - & - & - & $\mathrm{CF}$ & - & - & - & $\mathrm{CF}$ & - \\
\hline & & Boehmite & $\mathrm{CF}$ & - & - & - & $\mathrm{CF}$ & - & - & - & $\mathrm{CF}$ & - \\
\hline & \multirow{4}{*}{ Chromate } & As cleaned & DF & AZ31B & - & - & $\mathrm{DF}$ & AZ31B & - & - & $\mathrm{DF}$ & AZ31B \\
\hline & & Chromate-phosphate & DF & AZ31B & - & - & DF & AZ31B & - & - & $\mathrm{DF}$ & AZ31B \\
\hline & & Manganese & $\mathrm{DF}$ & AZ31B & - & - & $\mathrm{DF}$ & AZ31B & - & - & $\mathrm{DF}$ & AZ31B \\
\hline & & Boehmite & DF & AZ31B & - & - & $\mathrm{DF}$ & AZ31B & - & - & DF & AZ31B \\
\hline & \multirow{4}{*}{$\mathrm{Mg}(\mathrm{OH})_{2}$ coating } & As cleaned & $\mathrm{CF}$ & - & - & - & $\mathrm{CF}$ & - & - & - & $\mathrm{CF}$ & - \\
\hline & & Chromate-phosphate & $\mathrm{CF}$ & - & - & - & $\mathrm{CF}$ & - & - & - & $\mathrm{CF}$ & - \\
\hline & & Manganese & $\mathrm{CF}$ & - & - & - & $\mathrm{CF}$ & - & - & - & $\mathrm{CF}$ & - \\
\hline & & Boehmite & $\mathrm{CF}$ & - & - & - & $\mathrm{CF}$ & - & - & - & $\mathrm{CF}$ & - \\
\hline
\end{tabular}


Table 5 Failure patterns with various conversion coatings on AZ31B magnesium alloy - A6022 aluminum alloy. (Tensile lap-shear strength test)

\begin{tabular}{|c|c|c|c|c|}
\hline \multirow{2}{*}{$\begin{array}{l}\text { Epoxy } \\
\text { resin }\end{array}$} & \multicolumn{2}{|c|}{ Specimen } & \multirow{2}{*}{$\begin{array}{l}\text { Failure } \\
\text { patterns }\end{array}$} & \multirow{2}{*}{$\begin{array}{c}\text { Delamination } \\
\text { side }\end{array}$} \\
\hline & AZ31B & A6022 & & \\
\hline \multirow{12}{*}{$\begin{array}{c}\text { Conversion } \\
\text { coating }\end{array}$} & \multirow{4}{*}{ As cleaned } & As cleaned & $\mathrm{AF}$ & AZ31B \\
\hline & & Chromate-phosphate & DF & A6022 \\
\hline & & Manganese & DF & A6022 \\
\hline & & Boehmite & $\mathrm{AF}$ & AZ31B \\
\hline & \multirow{4}{*}{ Chromate } & As cleaned & DF & AZ31B \\
\hline & & Chromate-phosphate & $\mathrm{DF}$ & AZ31B \\
\hline & & Manganese & DF & AZ31B \\
\hline & & Boehmite & DF & AZ31B \\
\hline & \multirow{4}{*}{$\mathrm{Mg}(\mathrm{OH})_{2}$ coating } & As cleaned & $\mathrm{AF}$ & AZ31B \\
\hline & & Chromate-phosphate & DF & A6022 \\
\hline & & Manganese & DF & A6022 \\
\hline & & Boehmite & $\mathrm{AF}$ & AZ31B \\
\hline \multirow{2}{*}{$\begin{array}{l}\text { Acrylic } \\
\text { resin }\end{array}$} & \multicolumn{2}{|c|}{ Specimen } & Failure & Delamination \\
\hline & AZ31B & A6022 & patterns & side \\
\hline \multirow{12}{*}{$\begin{array}{c}\text { Conversion } \\
\text { coating }\end{array}$} & \multirow{4}{*}{ As cleaned } & As cleaned & $\mathrm{CF}$ & - \\
\hline & & Chromate-phosphate & $\mathrm{CF}$ & - \\
\hline & & Manganese & $\mathrm{CF}$ & - \\
\hline & & Boehmite & $\mathrm{CF}$ & - \\
\hline & \multirow{4}{*}{ Chromate } & As cleaned & $\mathrm{CF}$ & - \\
\hline & & Chromate-phosphate & $\mathrm{CF}$ & - \\
\hline & & Manganese & $\mathrm{CF}$ & - \\
\hline & & Boehmite & $\mathrm{CF}$ & - \\
\hline & \multirow{4}{*}{$\mathrm{Mg}(\mathrm{OH})_{2}$ coating } & As cleaned & $\mathrm{CF}$ & - \\
\hline & & Chromate-phosphate & $\mathrm{CF}$ & - \\
\hline & & Manganese & $\mathrm{CF}$ & - \\
\hline & & Boehmite & $\mathrm{CF}$ & - \\
\hline
\end{tabular}

(a)

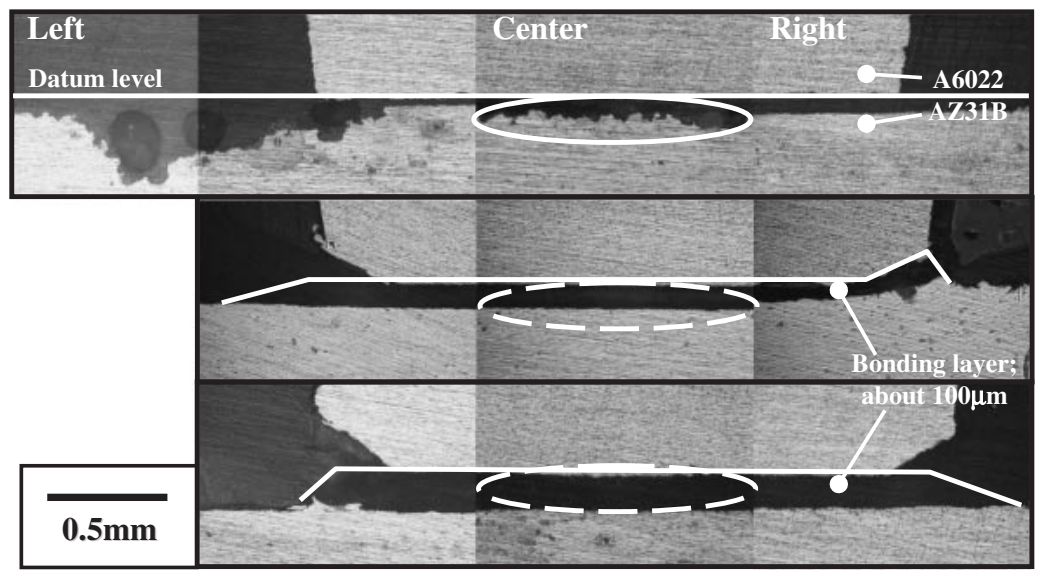

Fig. 10 Cross section images of galvanic corrosion test. (AZ31B As cleaned/A6022 As cleaned) (a) non-adhesive (b) epoxy resin (c) acrylic resin.

\section{REFERENCES}

1) S. Maeda: Nihon Parkerizing Technical Report 17 (2005) 10-19.

2) Y. Minamizaki: Journal of Japan Institute of Electronics packaging 6 (2003) 349-354.

3) M. Sekiguchi, C. Otani and M. Takaya: J.JILM 56 (2006)156-161.

4) Japanese Industrial Standards Committee; JIS K 5600-5-7, Adhesion test (Pull-off methods) (1999).

5) Japanese Industrial Standards Committee; JIS K 6850, Determination of tensile lap-shear strength of rigid-to-rigid bonded assemblies (1999).

6) Japanese Industrial Standards Committee; JIS K 6866, Designation of main failure patterns (1999).

7) Japanese Industrial Standards Committee; JIS Z 2371, Methods of salt spray testing (2000). 